\title{
Custom-Molded Offloading Footwear Effectively Prevents Recurrence and Amputation, and Lowers Mortality Rates in High-Risk Diabetic Foot Patients: A Multicenter, Prospective Observational Study
}

\author{
Xi Zhang, ', * Hongyan Wang, ', \\ Chenzhen Du,' Xiaoyun Fan,' \\ Long Cui, ${ }^{3}$ Heming Chen, ${ }^{4}$ Fang Deng, ${ }^{5}$ \\ Qiang Tong, ${ }^{6}$ Min He, ${ }^{7}$ Mei Yang, ${ }^{8}$ \\ Xingrong Tan, ${ }^{9}$ Lin Li, (iD ${ }^{10}$ \\ Zerong Liang, "' Yaqin Chen,' \\ Deqing Chen, ${ }^{3}$ David G Armstrong, ${ }^{14}$ \\ Wuquan Deng'
}

'Department of Endocrinology, Chongqing University Central Hospital, Chongqing University, Chongqing, People's Republic of China; ${ }^{2}$ Department of Endocrinology, Hospital for Occupational Diseases of Chongqing, Chongqing, People's Republic of China; ${ }^{3}$ Department of Endocrinology, Armed Police Hospital of Chongqing, Chongqing, People's Republic of China; ${ }^{4}$ Department of Endocrinology, Ankang Central Hospital, Ankang, Shaanxi, People's Republic of China; ${ }^{5}$ Department of Endocrinology, Chongqing Southwest Hospital, Chongqing, People's Republic of China; ${ }^{6}$ Department of Endocrinology, Chongqing Xinqiao Hospital, Chongqing, People's Republic of China; ${ }^{7}$ Department of Endocrinology, The People's Hospital of Shapingba District, Chongqing, People's Republic of China;

${ }^{8}$ Department of Endocrinology, The First People's Hospital of Chongqing Liangjiang New Area,

Chongqing, People's Republic of China;

'Department of Endocrinology, The 9th People's Hospital of Chongqing, Chongqing, People's Republic of China; ${ }^{10}$ Department of

Endocrinology, Zhejiang University School of Medicine Sir Run Run Shaw Hospital, Hangzhou, People's Republic of China; "Department of Endocrinology, Chongqing Red Cross Hospital, People's Hospital of Jiangbei District, Chongqing, 400020, People's Republic of China;

${ }^{12}$ Department of Endocrinology, The People's Hospital of Shizhu, Chongqing, People's Republic of China; ${ }^{13}$ Department of Endocrinology, The People's Hospital of Rongchang, Chongqing, People's Republic of China; ${ }^{14}$ Department of Surgery, Keck School of Medicine of the University of Southern California, Los Angeles, CA, USA

*These authors contributed equally to this work

Correspondence: Wuquan Deng

Email wuquandeng@cqu.edu.cn
Background: Recurrence of high-risk diabetic feet, after wound, healing is a common challenge among diabetic patients. Continuous use of an offloading device significantly prevents recurrence of high-risk diabetic feet, although patient adherence is imperative to ensuring this therapy's clinical efficacy. In this study, we explored clinical outcomes of patients with a high-risk diabetic foot who had been prescribed with custom-molded offloading footwear under different adherence conditions.

Methods: A total of 48 patients (17 females and 31 males) with high-risk diabetic feet, who had been with prescribed offloading footwear in 13 medical centers across 4 cities, were enrolled in the current study. The patients were assigned into either continuous offloading therapy (COT, $n=$ 31 ) or interrupted offloading therapy (IOT, $n=17$ ) groups, according to their adherence to the therapy. All patients were followed up monthly, and differences in recurrence, amputation, and deaths between the groups were analyzed at 4 months after therapy.

Results: Forty-eight patients met our inclusion criteria and were therefore included in the final analysis. Among them, 31 were stratified into the COT group and adhered to offloading therapy throughout the study period, whereas 17 were grouped as IOT and exhibited interrupted adherence to offloading therapy. We found statistically significant differences in recurrence rates ( 0 vs $38.46 \%, \mathrm{p}<0.01)$, amputation ( 0 vs $11.76 \%, \mathrm{p}<0.01)$, and deaths $(0 \%$ vs $5.88 \%, \mathrm{p}<0.01)$ between the groups during follow-up.

Conclusion: Patients' adherence is imperative to efficacy of custom-molded offloading footwear during treatment of high-risk diabetic foot. Further studies are needed to elucidate the role of improved design of the offloading device and the need for enhanced patient education for improved adherence.

Keywords: custom-molded offloading footwear, high-risk diabetic foot, patient adherence

\section{Introduction}

High-risk diabetic foot is prone to injury, infection and ulceration in diabetic patients. ${ }^{1}$ Previous studies have reported that the 1- and 5-year recurrence rates of high-risk diabetic feet $31.6 \%{ }^{2}$ and approximately $70 \%,{ }^{3}$ respectively. Refractory recurrence and slow healing rates have been linked to leg amputation and patient mortality. Moreover, highrisk diabetic feet have been associated with non-traumatic amputation, with more than $70 \%$ mortality rates within 5 years after amputation. ${ }^{4}$ Therefore, reducing the recurrence rate of high-risk diabetic feet can significantly improve amputation and lower mortality rates in this group of patients. 
High-risk diabetic foot recurrence is mainly attributed to abnormal lower limb movements and changes in plantar biomechanics. ${ }^{5-8}$ Chronic overload and friction to the feet, which are caused by wearing conventional shoes, can cause damage to the skin on the sole or dorsal foot, thereby exacerbating a patient's gait and balance ability over time. These changes ultimately lead to recurrence of foot ulcers and amputation. Soft tissues are prone to continuous overload, even after healing, a phenomenon that results in tissue necrosis and recurrence of high-risk diabetic foot. ${ }^{9}$ However, amputations and deaths can be prevented if patients receive appropriate care. ${ }^{10-12}$ According to the International Working Group on the Diabetic Foot (IWGDF), use of an offloading device can effectively prevent recurrence in high-risk diabetic foot patients. ${ }^{12}$ Moreover, this device can reduce plantar pressure on the painful part of the foot, by changing the center of gravity line, thereby improving body balance. Consequently, the device rationally distributes the pressure on the bottom of the foot and absorbs vibration, thus preventing recurrence of high-risk diabetic foot. ${ }^{13,14}$ Previous studies have demonstrated that continued use of the offloading device to improve plantar biomechanics and COT can effectively prevent recurrence of diabetic high-risk feet. ${ }^{15-18}$ Notably, patient adherence has been shown to play an important role in clinical outcomes during offloading therapy. ${ }^{19,20}$ In the present study, we explored the effects of patient adherence on diabetic foot recurrence, amputation, and mortality using offloading therapy in high-risk diabetic foot patients.

\section{Methods and Study Design}

A total of 87 patients, who were diagnosed with high-risk diabetic feet between January 1, 2018 and December 31, 2020, were enrolled at 13 medical centers across four cities in China. Inclusion criteria were adopted from the United States Medicare Guidelines. A patient was deemed eligible for the Medicare diabetic therapeutic footwear benefits if the treating physician provided a written statement certifying existence of at least one of the following conditions: 1) had undergone previous partial amputation of either foot; 2) had a history of previous foot ulceration on either foot; 3) had a history of pre-ulcerative calluses on either foot; 4) was diagnosed with peripheral neuropathy with evidence of callus formation on either foot; 5) presented with foot deformity; 6) presented with poor circulation on either foot; or 7) had a prescription for a particular type of foot wear from a physician.
Conversely, a participant was excluded if he or she 1) had a history of major amputations; 2) was diagnosed with neurologic or psychiatric disease; 3) was unable to walk without walking aids; 4) had visual impairment that was difficult to either treat or correct; 5) was diagnosed with unstable cardiovascular or unstable ischemic cerebrovascular diseases; (6) had severe renal dysfunction or severe liver dysfunction; 7) was diagnosed with a malignant tumor; 8) had osteoarticular diseases unrelated to diabetes; and 9) declined offloading therapy.

A total of 48 high-risk diabetic foot patients (17 females and 31 males) who were undergoing therapy with offloading device (Depth Inlay Shoes, Dave Med LLC, Chongqing, China) met the inclusion criteria and were ultimately recruited in the study. All patients were followed up monthly, through a face-to-face or telemedicine approach, because of COVID-19. At 4 months after offloading therapy, differences in recurrence, amputation, and mortality rates between the groups were determined and the effect of the offloading device evaluated. The definition of interrupted offloading therapy (IOT, $n=17$ ) group was based on discontinued offloading footwear therapy, for more than 4 weeks. Patients were divided into either continuous offloading therapy (COT, $n=31$ ) or IOT ( $n=17)$ groups, based on their level of adherence to offloading therapy. The patients' demographic information and clinical characteristics, including age, gender, height, BMI, diabetes duration, Wagner classification, diabetic neuropathy, diabetic retinopathy, foot deformity, vibrating perception threshold, history of amputation, plantar callus, peripheral artery disease, ischemia, osteoporosis, and methods of diabetes control were recorded and analyzed at the beginning of study. Patients were advised to wear the offloading device for 1-2 hours every day for the first week after discharge. Next, they were advised to wear the offloading device every day, if there did not experience any discomfort or foot grinding a week after discharge.

\section{Statistical Analysis}

Descriptive statistics, including proportions, means, and standard deviations were calculated. Differences between patients undergoing COT and those who presented with discontinuation of offloading therapy were determined using a $t$-test, while baseline data were analyzed using a Chi-square test. All statistical analyses were performed using SPSS 26 software (IBM, Armonk, NY). $P<0.05$ was considered statistically significant for all of the analyses. 


\section{Results}

A total of 48 , out of 88 , patients (including 17 women and 31 men) met our inclusion criteria and were included in the final analysis. A summary of the research design is presented using a flow chart in Figure 1. Results revealed no statistically significant differences in baseline data between the two groups (Table 1). Patients were fitted with molded offloading insole (Figure 2). A comparison of patients' main clinical outcomes between the two groups, 4 months after offloading therapy, revealed no DFU recurrence in 21 cases that previously had DFU, and none of the 31 patients the COT group had neither undergone amputation nor died during the study period. In contrast, the 13 patients with a history of DFU in the IOT group had a recurrence rate of $38.46 \%(5 / 13)$, with amputation and mortality rates of $11.76 \%$ and $5.88 \%$, respectively. We found statistically significant differences in recurrence, amputation and mortality rates between patients in the COT and IOT groups $(\mathrm{p}<0.01)$ (Figure 3 ).

\section{Discussion}

High-risk diabetic foot causes great pain and high economic burden to patients and their families. To manage the

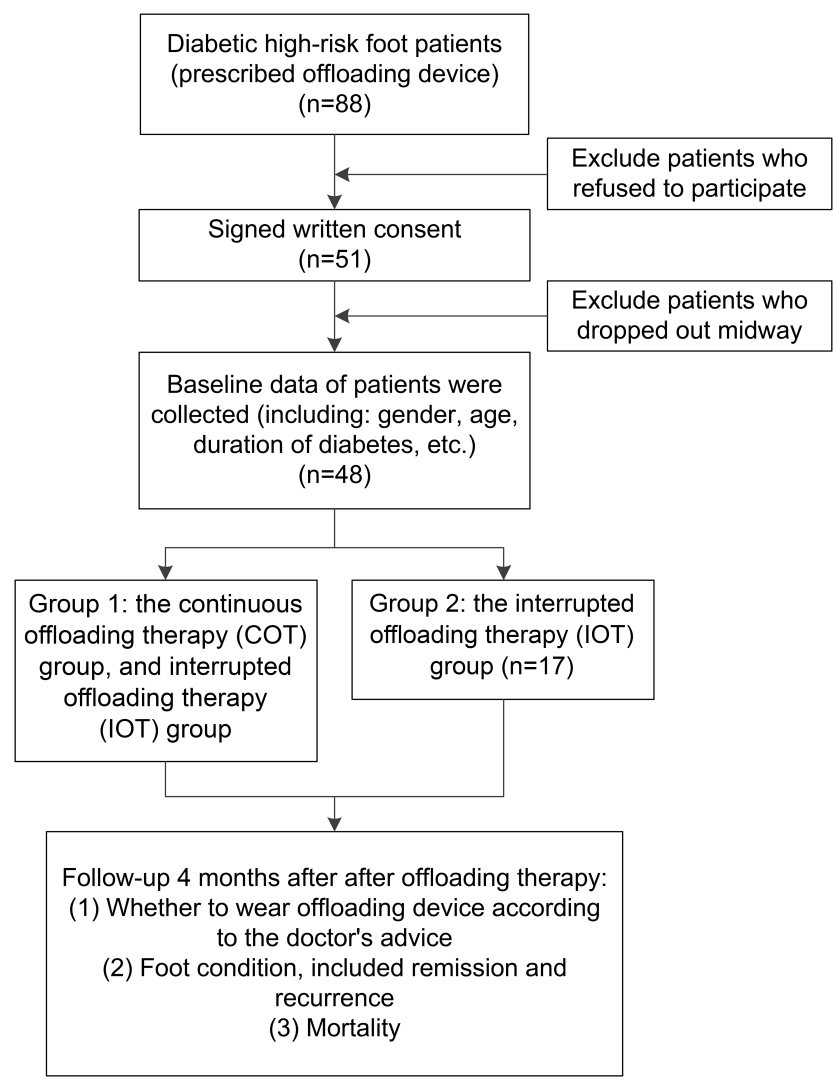

Figure I A flow chart describing the study design. condition, diabetic foot offloading footwear, comprising functional socks and custom insoles, has been used in some developed countries and is included in the medical insurance coverage. ${ }^{21}$ However, offloading device is not covered by medical insurance in most developing countries. Efficacy of using the offloading device for diabetic patients with high-risk foot for preventive treatment remains controversial. For example, Gao et al, ${ }^{22} \mathrm{Bus} \mathrm{et} \mathrm{al}^{23}$ and Maciejewski et $\mathrm{al}^{24}$ reported that the offloading device was effective in preventing recurrence of diabetic foot ulcers. In contrast, Macfarlane et $\mathrm{al}^{20}$ found no significant effects in use of the offloading device in high-risk diabetic feet patients. Notably, the authors attributed the lack of significant effects to low adherence.

Results from our previous study, in which we explored use of offloading device in neuropathic diabetic foot, revealed that the device changed plantar biomechanics using wearable sensor technology. ${ }^{8,25}$ Notably, this offloading therapy significantly improved clinical outcomes in these patients although one case who underwent IOT presented with recurrence during interruption of the therapy. ${ }^{8}$ In the current study, we further explored the effects of interrupting offloading therapy on recurrence and mortality rates in high-risk diabetic foot patients. To the best of our knowledge, this is the first multicenter study analyzing the use of offloading footwear therapy intervention in diabetic patients with high-risk foot. The findings from the present study, coupled with literature review, indicated that use of offloading device significantly reduced the risk of foot ulcer recurrence, amputation and mortality. ${ }^{26}$ In the current study, a total of 48 patients who were prescribed with the offloading device between 2018 and 2020 were selected for follow-up and exploration of the effect of patient based on clinical outcomes. Results showed that with high-risk diabetic foot patients with poor compliance to offloading therapy exhibited high recurrence, amputation, and mortality rates.

The poor adherence was attributed to several reasons: Firstly, patients were not willing to undergo the therapy. Secondly, the weight, appearance and comfort of the offloading device limited its use. ${ }^{27,28}$ Thirdly, some medical staff did not pay enough attention to the auxiliary and preventive treatment effects of the offloading device, while social awareness was low. Results from follow-up showed that some patients psychologically resisted offloading therapy. ${ }^{29,30}$ In addition, although clinicians recommended that the offloading device be worn for a long period, patients were worried about the associated 
Table I Baseline Characteristics of All Patients Participating in This Study

\begin{tabular}{|c|c|c|c|c|}
\hline Variables & All Patients $(n=48)$ & COT Group $(n=3 I)$ & IOT Group $(n=17)$ & P-value \\
\hline Age, years & $64.92 \pm 12.38$ & $62.77 \pm 11.50$ & $68.82 \pm 13.03$ & 0.106 \\
\hline Height, $\mathrm{cm}$ & $162.74 \pm 8.27$ & $|62.85 \pm 8.4|$ & $162.62 \pm 8.34$ & 0.933 \\
\hline BMI, $\mathrm{kg} / \mathrm{m}^{2}$ & $24.46 \pm 2.99$ & $24.35 \pm 2.45$ & $24.60 \pm 3.61$ & 0.804 \\
\hline Diabetes duration, years & $11.33 \pm 7.56$ & $11.91 \pm 7.57$ & $|0.59 \pm 7.7|$ & 0.595 \\
\hline \multicolumn{5}{|l|}{ Gender, \% } \\
\hline Female & $17(35.42)$ & $9(29.03)$ & $8(47.06)$ & 0.212 \\
\hline Male & $31(64.58)$ & $22(70.97)$ & $9(52.94)$ & \\
\hline \multicolumn{5}{|l|}{ Wagner classification, \% } \\
\hline $0-3$ & $36(75.00)$ & $24(77.42)$ & $12(70.59)$ & 0.862 \\
\hline $4-5$ & $12(25.00)$ & $7(22.58)$ & $5(29.4 I)$ & \\
\hline \multicolumn{5}{|l|}{ Diabetic retinopathy, \% } \\
\hline Yes & $23(47.92)$ & I7(54.84) & $6(35.29)$ & 0.195 \\
\hline No & $25(52.08)$ & $14(45.16)$ & II (64.7I) & \\
\hline \multicolumn{5}{|l|}{ Foot deformity, \% } \\
\hline Yes & $16(33.33)$ & $10(32.26)$ & $6(35.29)$ & 0.831 \\
\hline No & $32(66.67)$ & $21(67.74)$ & II (64.7I) & \\
\hline \multicolumn{5}{|l|}{ VPT>25volt, \% } \\
\hline Yes & $18(37.50)$ & $12(38.7 \mid)$ & 6(35.29) & 0.815 \\
\hline No & $30(62.50)$ & $19(61.29)$ & II (64.7I) & \\
\hline \multicolumn{5}{|l|}{ History of DFU, \% } \\
\hline Yes & $34(70.83)$ & $21(67.74)$ & $13(76.47)$ & $0.74 I$ \\
\hline No & $14(29.17)$ & $10(32.26)$ & $4(23.53)$ & \\
\hline \multicolumn{5}{|l|}{ History of amputation, \% } \\
\hline Yes & $16(33.33)$ & $8(25.8 I)$ & $8(47.06)$ & 0.135 \\
\hline No & $32(66.67)$ & $23(74.19)$ & $9(52.94)$ & \\
\hline \multicolumn{5}{|l|}{ Plantar callus, $\%$} \\
\hline Yes & $20(41.67)$ & $14(45.16)$ & 6(35.29) & 0.507 \\
\hline No & $28(58.33)$ & $17(54.84)$ & II (64.7I) & \\
\hline \multicolumn{5}{|l|}{ Diabetic neuropathy, \% } \\
\hline Yes & $42(87.50)$ & $27(87.10)$ & $15(88.24)$ & I \\
\hline No & $6(12.50)$ & $4(12.90)$ & $2(11.76)$ & \\
\hline \multicolumn{5}{|l|}{ PAD, $\%$} \\
\hline Yes & $23(47.92)$ & $13(41.94)$ & $10(58.82)$ & 0.263 \\
\hline No & $25(52.08)$ & $18(58.06)$ & $7(41.18)$ & \\
\hline \multicolumn{5}{|l|}{ Ischemia, \% } \\
\hline Yes & $31(64.58)$ & $18(58.06)$ & $13(76.47)$ & 0.202 \\
\hline No & $17(35.42)$ & $13(4 \mid .94)$ & $4(23.53)$ & \\
\hline Osteoporosis, \% & & & & 0.263 \\
\hline Yes & $25(52.08)$ & $18(58.06)$ & $7(41.18)$ & \\
\hline No & $23(47.92)$ & $13(41.94)$ & $10(58.82)$ & \\
\hline \multicolumn{5}{|l|}{ Methods of blood glucose control, \% } \\
\hline Insulin & $2(4.16)$ & $\mathrm{I}(3.22)$ & $\mathrm{I}(5.88)$ & \\
\hline Anti-hyperglycemic drugs & $20(41.67)$ & $13(4 \mid .94)$ & $7(41.18)$ & 0.907 \\
\hline Anti-hyperglycemic drugs and Insulin & $26(54.17)$ & $17(54.84)$ & $9(52.94)$ & \\
\hline
\end{tabular}

Abbreviations: COT, continuous offloading therapy; IOT, interrupted offloading therapy; BMI, body Mass Index; VPT, vibrating perception threshold; DFU, diabetic foot ulcer; PAD, peripheral artery disease.

expenses. In addition, the patients had a negative perception of the device, mainly because wearing it implied that they had not fully recovered thus increasing the psychological burden. Although the offloading device is efficacious in preventing amputation and mortality in patients at high risk of diabetic foot, only a handful of clinicians and researchers have described its role.

Improving patient adherence to use of the offloading device can significantly improve clinical outcomes of this therapy in diabetic foot. This adherence may be improved by 

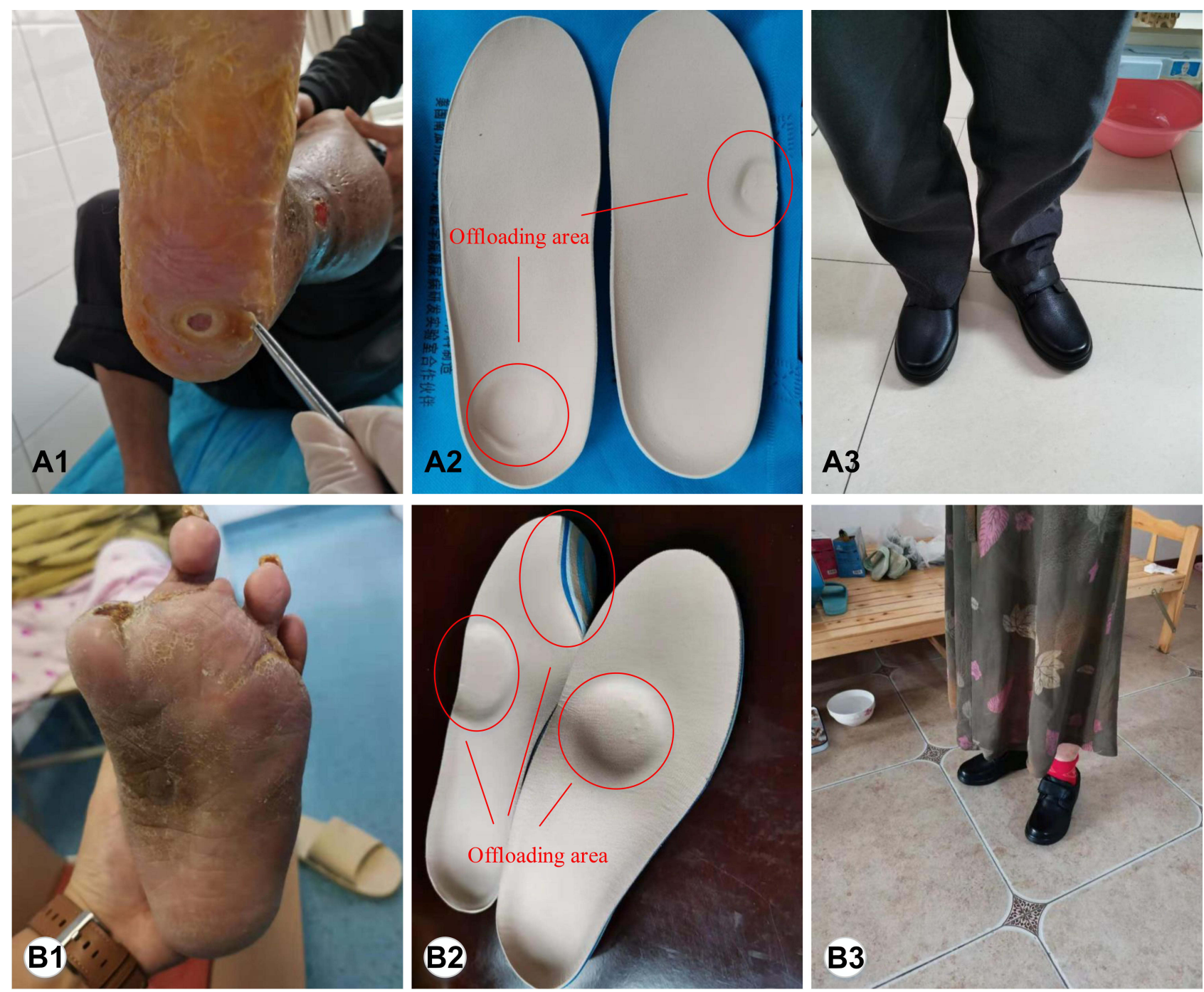

Figure 2 Profiles of patients' foot conditions and offloading therapy. Patient A: Recurrent neuropathic plantar foot ulcer on the left heel and the fifth right plantar metatarsophalangeal joint (AI). Personalized offloading insoles (A2). Wearing of the offloading footwear (A3). Patient B: Recurrent neuropathic plantar foot ulcer on the right foot and amputation of the first and third toes of the left foot (B I). Personalized offloading and orthopedic insoles (B2). Wearing the offloading footwear (B3).

providing patients with more color choices, as well as styles and materials. Health facilities and clinicians should also increase awareness of different approaches for treatment

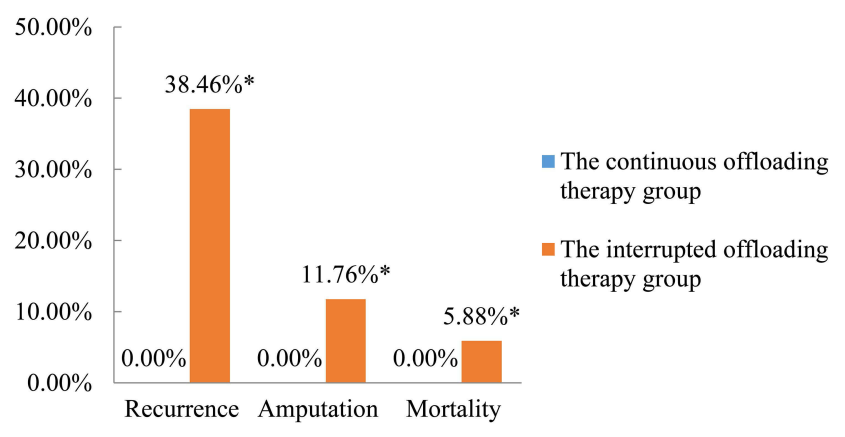

Figure 3 Clinical outcomes of patients included in the study. *There were statistically significant difference in recurrence, amputation and mortality rates between patients in the continuous offloading therapy and those $e$ in the interruption offloading therapy groups $(\mathrm{P}<0.0 \mathrm{I})$. and disease prevention, while patients and medical staff should undergo health education to equip them with information regarding the positive effects of offloading therapy. ${ }^{31} \mathrm{We}$ hypothesize that increased health education for patients and medical staff will improve the level of foot self-care knowledge and patient behavior towards the therapy. Furthermore, medical personnel should undergo regular training on prevention and adjuvant treatment of diabetic foot using offloading device to improve their effectiveness in providing care for patients with high-risk foot.

Results of the present study further confirm efficacy of the offloading device as an adjuvant therapy for patients with diabetic high-risk foot. This is because wearing an offloading device significantly lowers the number of patients with high-risk diabetes feet, and subsequently reduces the socioeconomic burden, risk of infections, 
amputation, and death of patients associated with this condition.

This study had a few limitations. Firstly, our sample size was relatively small. Future studies should enroll more patients to establish barriers regarding continuous use of offloading footwear and help improve diabetesrelated foot ulcers. Secondly, the follow-up was short due to the COVID-19 pandemic.

\section{Conclusion}

Findings for the present study showed that the offloading device is an efficacious auxiliary therapeutic strategy for prevention of recurrence and amputation, and lowering mortality rates in diabetic foot patients. Notably, patient adherence is imperative to efficacy of this therapy.

\section{Data Sharing Statement}

The data are available on reasonable request from the corresponding author (wuquandeng@cqu.edu.cn).

\section{Ethics Approval and Informed Consent}

The study complied with the Declaration of Helsinki. The study, named Footwear and Offloading Optimum Therapy (FOOT) study, was approved by the institutional review board (IRB) of Chongqing University Central Hospital (ChiCTR1900022468). All protocols were conducted in accordance with the declaration of Helsinki. All participants provided written informed consent to participate in the study.

\section{Acknowledgments}

We thank all the participants in the present study for their participation and valuable feedbacks. This research was funded by the Joint Medical Research Programs of Chongqing Science and Technology Bureau and Health Commission Foundation (Grant No. 2020GDRC023), the Science and Technology Research Program of Chongqing Municipal Education Commission (Grant No. KJQN201900101), the Natural Science Foundation of Chongqing Municipal Science and Technology Bureau (cstc2020jcyj-msxmX0298). This study is partially supported by National Institutes of Health, National Institute of Diabetes and Digestive and Kidney Diseases Award Number 1R01124789-01A1 and National Science Foundation (NSF) Center to Stream Healthcare in Place
(\#C2SHiP) CNS Award Number 2052578 awarded to Prof. Armstrong DG.

\section{Disclosure}

The authors report no conflicts of interest in this work.

\section{References}

1. Boulton AJ. The diabetic foot: from art to science. The 18th Camillo Golgi lecture. Diabetologia. 2004;47(8):1343-1353. doi:10.1007/ s00125-004-1463-y

2. Jiang Y, Wang X, Xia L, et al. A cohort study of diabetic patients and diabetic foot ulceration patients in China. Wound Repair Regen. 2015;23(2):222-230. doi:10.1111/wrr.12263

3. Turns M. The diabetic foot: an overview for community nurses. $\mathrm{Br}$ J Community Nurs. 2012;17(9):422,424-427,430-433. doi:10.12968/ bjen.2012.17.9.422

4. Armstrong DG, Boulton AJM, Bus SA. Diabetic foot ulcers and their recurrence. $N$ Engl J Med. 2017;376(24):2367-2375. doi:10.1056/ NEJMra1615439

5. Arts ML, Waaijman R, De Haart M, et al. Offloading effect of therapeutic footwear in patients with diabetic neuropathy at high risk for plantar foot ulceration. Diabet Med. 2012;29 (12):1534-1541. doi:10.1111/j.1464-5491.2012.03770.x

6. Bus SA. Priorities in offloading the diabetic foot. Diabetes Metab Res Rev. 2012;28(Suppl 1):54-59. doi:10.1002/dmrr.2240

7. Deng W, Deng F, Chen B, et al. Association between peripheral neuropathy and biomechanics performance in patients with type 2 diabetes mellitus. Diabetes. 2019:68. doi:10.2337/db19-626-P.

8. Du C, Wang H, Chen H, et al. The feasibility and effectiveness of wearable sensor technology in the management of elderly diabetics with foot ulcer remission: a proof-of-concept pilot study with six cases. Gerontology. 2021;67(4):493-502. doi:10.1159/000513729

9. Van Netten JJ, Raspovic A, Lavery LA, et al. Prevention of foot ulcers in the at-risk patient with diabetes: a systematic review. Diabetes Metab Res Rev. 2020;36(Suppl 1):e3270. doi:10.1002/dmrr.3270

10. Lazzarini PA, Pacella RE, Armstrong DG, et al. Diabetes-related lower-extremity complications are a leading cause of the global burden of disability. Diabet Med.2018;35(9):1297-1299. doi:10.1111/dme.13680

11. Pickwell KM, Siersma VD, Kars M, et al. Diabetic foot disease: impact of ulcer location on ulcer healing. Diabetes Metab Res Rev. 2013;29(5):377-383. doi:10.1002/dmrr.2400

12. Bus SA, Van Netten JJ, Lavery LA, et al. IWGDF guidance on the prevention of foot ulcers in at-risk patients with diabetes. Diabetes Metab Res Rev. 2016;32(Suppl 1):16-24. doi:10.1002/dmrr.2696

13. Waaijman R, De Haart M, Arts ML, et al. Risk factors for plantar foot ulcer recurrence in neuropathic diabetic patients. Diabetes Care. 2014;37(6):1697-1705. doi:10.2337/dc13-2470

14. Ahmed S, Barwick A, Butterworth P, et al. Footwear and insole design features that reduce neuropathic plantar forefoot ulcer risk in people with diabetes: a systematic literature review. $J$ Foot Ankle Res. 2020;13(1):30. doi:10.1186/s13047-020-00400-4

15. Ha Van G, Siney H, Hartmann-Heurtier A, et al. Nonremovable, windowed, fiberglass cast boot in the treatment of diabetic plantar ulcers: efficacy, safety, and compliance. Diabetes Care. 2003;26 (10):2848-2852. doi:10.2337/diacare.26.10.2848

16. Waaijman R, Keukenkamp R, De Haart M, et al. Adherence to wearing prescription custom-made footwear in patients with diabetes at high risk for plantar foot ulceration. Diabetes Care. 2013;36 (6):1613-1618. doi:10.2337/dc12-1330

17. Chantelau E, Haage P. An audit of cushioned diabetic footwear: relation to patient compliance. Diabet Med. 1994;11(1):114-116. doi:10.1111/j.1464-5491.1994.tb00240.x 
18. Hingorani A, Lamuraglia GM, Henke P, et al. The management of diabetic foot: a clinical practice guideline by the Society for Vascular Surgery in collaboration with the American Podiatric Medical Association and the Society for Vascular Medicine. J Vasc Surg. 2016;63(2Suppl):3S-21S. doi:10.1016/j.jvs.2015.10.003

19. Jarl G, Alnemo J, Tranberg R, et al. Gender differences in attitudes and attributes of people using therapeutic shoes for diabetic foo complications. J Foot Ankle Res. 2019;12(1):21. doi:10.1186/ s13047-019-0327-0

20. Macfarlane DJ, Jensen JL. Factors in diabetic footwear compliance. J Am Podiatr Med Assoc. 2003;93(6):485-491. doi:10.7547/87507315-936-485

21. Wooldridge J, Bergeron J, Thornton C. Preventing diabetic foot disease: lessons from the Medicare therapeutic shoe demonstration. Am J Public Health. 1996;86(7):935-938. doi:10.2105/AJPH.86.7.935

22. Gao Y, Wang C, Chen D, et al. Effects of novel diabetic therapeutic footwear on preventing ulcer recurrence in patients with a history of diabetic foot ulceration: study protocol for an open-label, randomized, controlled trial. Trials. 2021;22(1):151. doi:10.1186/s13063-021-05098-8

23. Bus SA, Waaijman R, Arts M, et al. Effect of custom-made footwear on foot ulcer recurrence in diabetes: a multicenter randomized controlled trial. Diabetes Care. 2013;36(12):4109-4116. doi:10.2337/ dc13-0996

24. Maciejewski ML, Reiber GE, Smith DG, et al. Effectiveness of diabetic therapeutic footwear in preventing reulceration. Diabetes Care. 2004;27(7):1774-1782. doi:10.2337/diacare.27.7.1774
25. Jiang X, Li N, Yuan Y, et al. Limb salvage and prevention of ulcer recurrence in a chronic refractory diabetic foot osteomyelitis. Diabetes Metab Syndr Obes. 2020;13:2289-2296. doi:10.2147/DMSO.S254586

26. Bus SA, Haspels R, Busch-Westbroek TE. Evaluation and optimization of therapeutic footwear for neuropathic diabetic foot patients using in-shoe plantar pressure analysis. Diabetes Care. 2011;34 (7):1595-1600. doi:10.2337/dc10-2206

27. Johnson M, Newton P, Goyder E. Patient and professional perspectives on prescribed therapeutic footwear for people with diabetes: a vignette study. Patient Educ Couns. 2006;64(1-3):167-172. doi:10.1016/j.pec.2005.12.013

28. Paton JS, Roberts A, Bruce GK, et al. Patients' experience of therapeutic footwear whilst living at risk of neuropathic diabetic foot ulceration: an interpretative phenomenological analysis (IPA). J Foot Ankle Res. 2014;7(1):16. doi:10.1186/1757-1146-7-16

29. Vileikyte L, Rubin RR, Leventhal H. Psychological aspects of diabetic neuropathic foot complications: an overview. Diabetes Metab Res Rev. 2004;20(Suppl 1):S13-8. doi:10.1002/dmrr.437

30. Beattie AM, Campbell R, Vedhara K. What ever I do it's a lost cause. The emotional and behavioural experiences of individuals who are ulcer free living with the threat of developing further diabetic foot ulcers: a qualitative interview study. Health Expect. 2014;17 (3):429-439. doi:10.1111/j.1369-7625.2012.00768.x

31. Jiang X, Yuan Y, Ma Y, et al. Pain management in people with diabetes-related chronic limb-threatening ischemia. J Diabetes Res. 2021;2021:6699292. doi:10.1155/2021/6699292

Diabetes, Metabolic Syndrome and Obesity: Targets and Therapy

Dovepress

\section{Publish your work in this journal}

Diabetes, Metabolic Syndrome and Obesity: Targets and Therapy is an international, peer-reviewed open-access journal committed to the rapid publication of the latest laboratory and clinical findings in the fields of diabetes, metabolic syndrome and obesity research. Original research, review, case reports, hypothesis formation, expert opinion and commentaries are all considered for publication. The manuscript management system is completely online and includes a very quick and fair peer-review system, which is all easy to use. Visit http://www.dovepress.com/testimonials.php to read real quotes from published authors.

Submit your manuscript here: https://www.dovepress.com/diabetes-metabolic-syndrome-and-obesity-targets-and-therapy-journal 